Accepted on 2015 September 23 for the publication of ApJ

\title{
Origin of Interplanetary Dust through Optical Properties of Zodiacal Light
}

\author{
Hongu Yang ${ }^{1}$, Masateru Ishiguro ${ }^{1}$ \\ ${ }^{1}$ Department of Physics and Astronomy, Seoul National University, 599 Gwanak-ro, \\ Gwanak-gu, Seoul 151-742, Republic of Korea \\ hongu@astro.snu.ac.kr \\ ishiguro@astro.snu.ac.kr
}

\begin{abstract}
This study investigates the origin of interplanetary dust particles (IDPs) through the optical properties, albedo and spectral gradient, of zodiacal light. The optical properties were compared with those of potential parent bodies in the solar system, which include D-type (as analogue of cometary nuclei), Ctype, S-type, X-type, and B-type asteroids. We applied Bayesian inference on the mixture model made from the distribution of these sources, and found that $>90 \%$ of the interplanetary dust particles originate from comets (or its spectral analogues, D-type asteroids). Although some classes of asteroids (C-type and $\mathrm{X}$-type) may make a moderate contribution, ordinary chondrite-like particles from S-type asteroids occupy a negligible fraction of the interplanetary dust cloud complex. The overall optical properties of the zodiacal light were similar to those of chondritic porous IDPs, supporting the dominance of cometary particles in zodiacal cloud.
\end{abstract}

Subject headings: zodiacal dust; interplanetary medium; comets: general; asteroids: general; interstellar dust

\section{Introduction}

The purpose of this study is to investigate the origin of interplanetary dust particles (IDPs) taking into account the optical similarities and diversities between zodiacal light and 
reflections from minor bodies in the solar system, such as comets and asteroids. An enormous number of IDPs are distributed in interplanetary space. They are observable as scattered sunlight in the optical wavelength (zodiacal light) and as thermal radiation in the mid- and far-infrared wavelengths (zodiacal emission). The IDPs cloud, occasionally referred to as a zodiacal cloud, erodes on a time scale of $10^{3}-10^{7}$ years (depending on the size and orbit, $<1 / 100$ of the age of the solar system) due to Poynting-Robertson drag, mutual collisions among the IDPs, and planetary perturbations (Mann et al. 2006; Gor'kavyi et al. 1997; Dikarev et al. 2001). The mass-loss rate around the Earth's orbit is estimated to be $\approx 10^{3}$ $\mathrm{kg} \mathrm{s}^{-1}$ (Grun et al. 1985; Mann \& Czechowski 2005). It is, therefore, natural to think that ongoing dust production, such as impacts or ice sublimation, is compensating for the erosion of the zodiacal cloud.

The origins of IDPs have been studied through the spatial distribution of the zodiacal light. Early research for explaining the spatial distribution expected large contribution from the asteroidal origin IDPs (see e.g. Dermott et al. (1996)). Later, Hahn et al. (2002) compared the surface brightness distribution of zodiacal light, taken with the Clementine spacecraft onboard camera, to the inclination distributions of comets and asteroids and suggested that a significant fraction of dust particles at 1 AU are of cometary origin. Nesvorný et al. (2010) further performed a numerical simulation for dust particles ejected from six different orbital groups (asteroid families, main belt asteroids, Jupiter family comets (JFCs), dormant JFCs, Halley-type comets, and Oort cloud comets), and compared the brightness distribution of the modeled zodiacal emission to that of zodiacal emission observed by an infrared space telescope. They suggested that $85 \%-95 \%$ of IDPs observable as zodiacal emission originate from JFCs.

Although these recent studies on the brightness distribution favor cometary sources, little is known about the origin of IDPs in terms of their optical properties. Recently, Ishiguro et al. (2013) derived the geometric albedo of IDPs by comparing the brightness of the Gegenschein, a part of the zodiacal light enhanced by backward scattering enhancement, to the infrared model (Kelsall et al. 1998). The research provided possibilities to study the origin of IDPs from a different aspect than previous studies. In this study, we considered the origin of IDPs through the comparison of the albedo and spectral gradient of zodiacal light with those of the potential parent bodies, and present a discussion based on previous studies. 


\section{Methodology}

The size distribution of IDPs was studied through lunar microcrater counting and in situ flux measurements (Grun et al. 1985; Divine 1993). These studies suggested that the effective cross section of IDPs around the Earth's orbit is dominated by large $(10-100 \mu \mathrm{m})$ particles. The opposition effect found in Gegenschein supports the idea that IDPs, which make up the zodiacal light. are significantly larger than the optical wavelength (Ishiguro et al. 2013; Buffington et al. 2009). Accordingly, we could assume that the optical properties of IDPs are similar to those of big objects, such as comets and asteroids. We thus postulated the albedo $(\mathrm{A})$ and the spectral gradient $\left(S^{\prime}\right)$ of the IDPs according to those of potential dust sources in the following discussion.

\subsection{Albedo and spectral gradient of zodiacal light}

The albedo of IDPs has been measured using several methods. Hanner (1980) compared the zodiacal light brightness to the IDPs model derived from the Lunar microcrater records. Lumme \& Bowell (1985) derived the albedo value of IDPs, which can explain the polarization distribution of zodiacal light. Dumont \& Levasseur-Regourd (1988) compared the optical and infrared brightness at the solar elongation of $90^{\circ}$. Recently, Ishiguro et al. (2013) directly measured the geometric albedo of zodiacal light by comparing the optical and infrared (Kelsall et al. 1998) flux at the anti-solar point. They deduced that the albedo of the smooth zodiacal light component is $A=0.06 \pm 0.01$, after subtracting weak fine-scale features associated with asteroidal collisional families, namely, asteroidal dust bands. In this paper, we adopted the albedo value in Ishiguro et al. (2013).

Small bodies in the solar system generally show linear spectra in a range around 4500$7500 \AA$. It is useful to express the spectral index using the normalized reflectivity gradient, $S^{\prime}\left[\begin{array}{lll}\% & 10^{-3} & \AA\end{array}\right]$ :

$$
S^{\prime}=\frac{1}{\bar{S}} \frac{d S}{d \lambda},
$$

where $S$ is the reflectance, defined as the flux density of an object divided by the flux density of the Sun at the wavelength $\lambda$, and $\bar{S}$ and $d S / d \lambda$ denote the average reflectance and spectral gradient in the wavelength range, respectively. The spectra of zodiacal light have been measured mostly at infrared wavelengths from space (Matsuura et al. 1995: Matsumoto et al. 1996; Fixsen \& Mather 2002; Reach et al. 2003; Ootsubo et al. 2009; Tsumura et al. 2010). Since there is no spectrographic data available in the optical wavelength range, we derived the optical spectral gradient $S^{\prime}$ by a log-linear fitting using compiled photometric data taken at different wavelengths around $4600 \AA$ (Leinert et al. 1998). The regression 
formula for the ratio between the solar spectrum and the zodiacal light spectrum is given by,

$$
I_{\lambda} \propto\left[1.0+0.9 \times \log \left(\frac{\lambda}{5000 \AA}\right)\right] I_{\odot}
$$

where $I_{\lambda}$ and $I_{\odot}$ denote the flux densities of zodiacal light and the Sun at wavelength $\lambda$, respectively. Eq. (2) is applicable for zodiacal light in the spectral range of $\lambda \leq 5000 \AA$ at a solar elongation $>90^{\circ}$ (Leinert et al. 1998). From Eq. (2), we obtained the spectral gradient of IDPs as $S^{\prime}=8.5 \pm 1.0 \% \cdot 1000 \AA^{-1}$ at $4600 \AA$. Note that we derived $S^{\prime}$ at $4600 \AA$ in order to match the measured wavelength of the albedo (Ishiguro et al. 2013).

\subsection{Data sources}

Turning now to the IDPs sources, we assumed that they originate from asteroids and comets. In addition, some IDPs may originate from interstellar space (Hahn et al. 2002). For asteroids, we considered five major taxonomic types, namely $\mathrm{C}_{-}^{-}, \mathrm{X}-, \mathrm{S}-, \mathrm{B}-$ and $\mathrm{D}-$ types (DeMeo \& Carry 2013), as input data. Since the optical properties of cometary nuclei are similar to those of D-type asteroids (one taxonomic type of asteroids), we do not discriminate D-type asteroids from cometary nuclei. We thus assumed that IDPs consist of dust particles from six populations: C-type, X-type, S-type, and B-type asteroids as representatives of asteroids, cometary nuclei (including D-type asteroids), and interstellar dust.

To create a template of the optical properties of six potential dust sources, we made use of catalogs of albedos and spectra of asteroids and comets. For asteroids, we used the Asteroid Catalog Using AKARI(AcuA) catalog as a dataset of albedos (Usui et al. 2011), and the SMASSII catalog as datasets of spectral gradients (Bus 1999; Bus \& Binzel 2002b; Binzel et al. 2004). We found 274 C-type, 222 S-type, 191 X-type, 40 B-type and 33 D-type asteroids archived in both catalogs. For C-type, B-type and D-type asteroids, which show no obvious absorption, we used the spectral gradient values measured between $4350 \AA$ and 9250A (Bus \& Binzel 2002b; Binzel et al. 2004). For the S-type and X-type asteroids, which may have an absorption band around $>7000 \AA$, we used the data at $4400 \AA-7000 \AA$ (Bus 1999). Albedos and spectral gradients of 10 cometary nuclei were compiled from various previous studies, shown in Table 1. For interstellar dust, we used the optical properties of average galactic dust particles at $4600 \AA$, that is, $A=0.67$ and $S^{\prime}=-23 \pm 1 \% \cdot 1000 \AA^{-1}$ (Draine 2003). We ignored some taxonomic types of asteroids, such as K-type, L-type and $\mathrm{V}$-type as discussed in Section 4.

Figure 1 shows the relationship between the albedos and spectral gradients for the IDPs and the potential parent bodies described above. In the diagram, the datum of IDPs is 
Table 1. Optical properties of cometary nuclei.

\begin{tabular}{ccccc}
\hline \hline Name & type & albedo & spectral gradient & references \\
\hline 1P/Halley & Halley type & 0.043 & 7.5 & $\mathrm{a}, \mathrm{c}, \mathrm{g}$ \\
2P/Encke & Encke type & 0.050 & 11 & $\mathrm{~h}, \mathrm{j}, \mathrm{r}$ \\
9P/Tempel 1 & Jupiter family & 0.056 & 12.5 & $\mathrm{q}$ \\
10P/Tempel 2 & Jupiter family & 0.022 & 20 & $\mathrm{e}, \mathrm{f}$ \\
28P/Neujmin 1 & Jupiter family & 0.025 & 11.8 & $\mathrm{~b}, \mathrm{k}, \mathrm{p}$ \\
49P/Arend-Rigaux & Jupiter family & 0.028 & 10.4 & $\mathrm{~d}, \mathrm{i}$ \\
67P/Churyumov-Gerasimenko & Jupiter family & 0.047 & 10 & $\mathrm{o}, \mathrm{s}, \mathrm{t}, \mathrm{u}$ \\
103P/Hartley 2 & Jupiter family & 0.045 & 8.1 & $\mathrm{v}$ \\
162P/Siding Spring & Jupiter family & 0.034 & 9.2 & $\mathrm{~m}, \mathrm{n}$ \\
$\mathrm{C} / 2001$ OG $_{108}$ (LONEOS) & Halley type & 0.040 & 9 & $\mathrm{l}$ \\
\hline
\end{tabular}

Note. - a - Sagdeev et al. (1986), b - Campins et al. (1987), c - Keller et al. (1987), d Millis et al. (1988), e - A'Hearn et al. (1989), f - Jewitt \& Luu (1989), g - Thomas \& Keller (1989), h - Luu \& Jewitt (1990). i - Luu (1993). i - Fernández et al. (2000). k - Campins \& Fernández (2002), l - Abell et al. (2005), m - Campins et al. (2006), n - Fernández et al. (2006). o - Lamv et al. (2006). p - Campins et al. (2007). a - Li et al. (2007). r - Boehnhardt et al. (2008), s - Lamy et al. (2008), t - Tubiana et al. (2008), u - Kelley et al. (2009), v Li et al. (2013) 
located within the population of comets and its spectral analog, D-type asteroids, suggesting that the major constituents are of cometary origin. In the following section, we will further investigate the contribution of each population using statistical analysis.

\subsection{Bayesian analysis}

When we chose objects from a type of population and calculated the correlation coefficients between the albedos and spectral gradients, the absolute values were as low as -0.14 , -0.04, 0.16, -0.36 and -0.11, for the C-type, S-type, X-type, B-type and D-type asteroids, respectively. Therefore, we considered these two properties, the albedo and spectral gradients, as being independent of each other. Within a population, we simply assumed that the albedo follows a log-normal distribution whereas the spectral gradient has a Gaussian distribution. We fit the distributions shown in Figure 1 to the model distributions shown in Figures 2 and 3. Because interstellar dust particles, which have limited contribution to IDPs, have optical properties that are very different from those of solar system objects, we assumed that the interstellar dust particles have a fixed albedo value of $A=0.673$ and a spectral

gradient of $S^{\prime}=-23.2 \pm 0.8 \% \cdot 1000 \AA^{-1}$, and did not consider its statistical distributions in the following analysis.

We made a $2 \%$ grid for the possible combinations of fractional contributions from the source populations. Then, through linearly combining the probability distributions of the source populations according to the given contribution, we could generate a mixture probability distribution for an optical property of a single dust particle. Different populations were weighted according to the average albedos. From the probability distribution for a single particle, we calculated the expected average values of both albedo and spectral gradient for the IDPs complex using Monte Carlo (MC) simulations. At the every grid point, a MC simulation with 500 sample particles was generated 3000 times. Under these conditions, the expected average values follow a Gaussian distribution, with a standard deviation of less than $10 \%$ of the uncertainty on the zodiacal light measurement. The probability of obtaining the measured optical properties was calculated at each grid point. By applying Bayesian inference with a flat prior, the probability was accepted as that of the assumed contribution of the grid point represent real situation. 


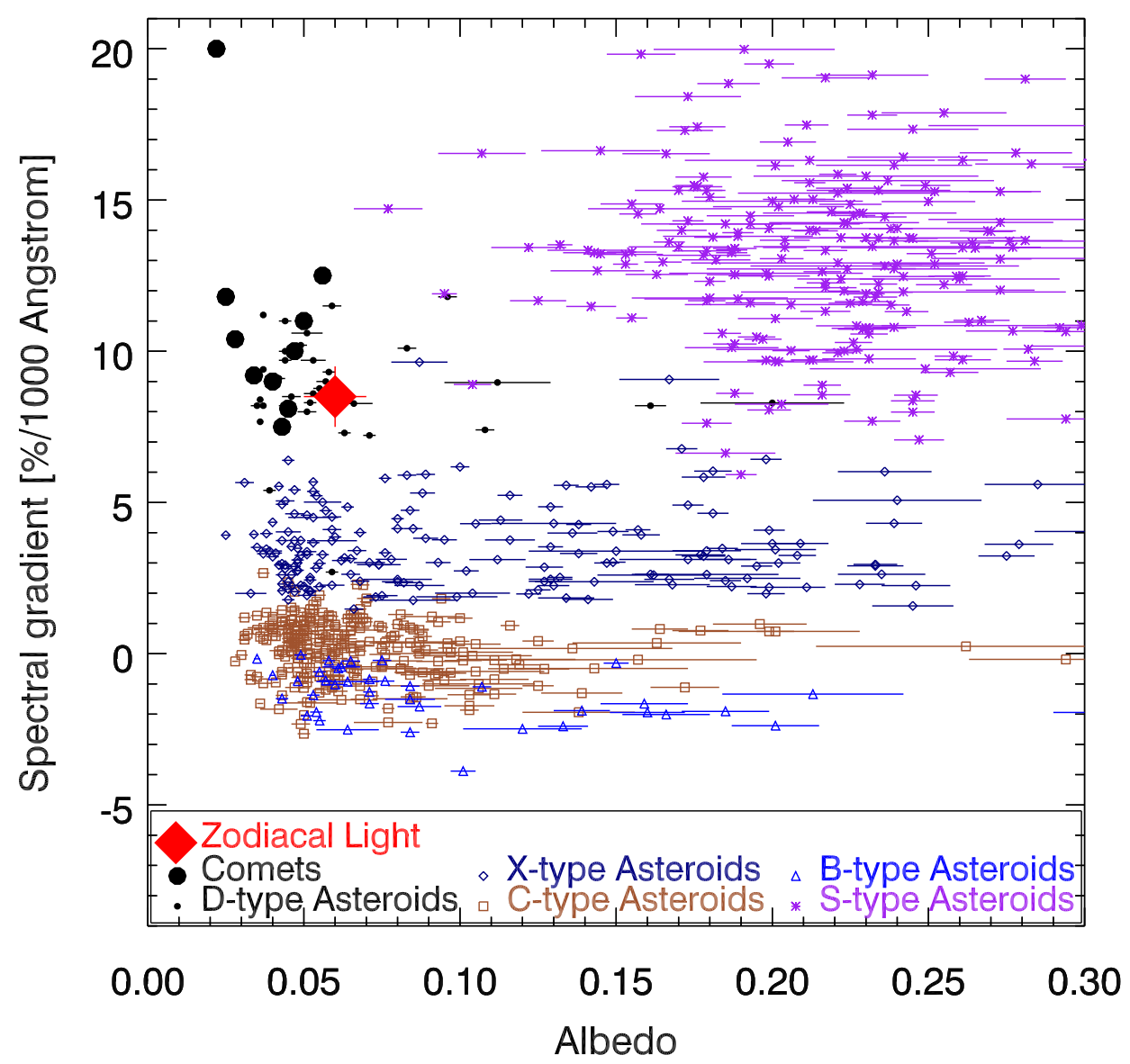

Fig. 1.- Spectral gradients $S^{\prime}$ with respect to albedos $A$ of asteroids, comets and zodiacal light. Uncertainties of albedos are appended in the plot. The $1 \sigma$ measurement uncertainties in spectral gradients are ordinarily about $0.7 \% \cdot 1000 \AA^{-1}$. 

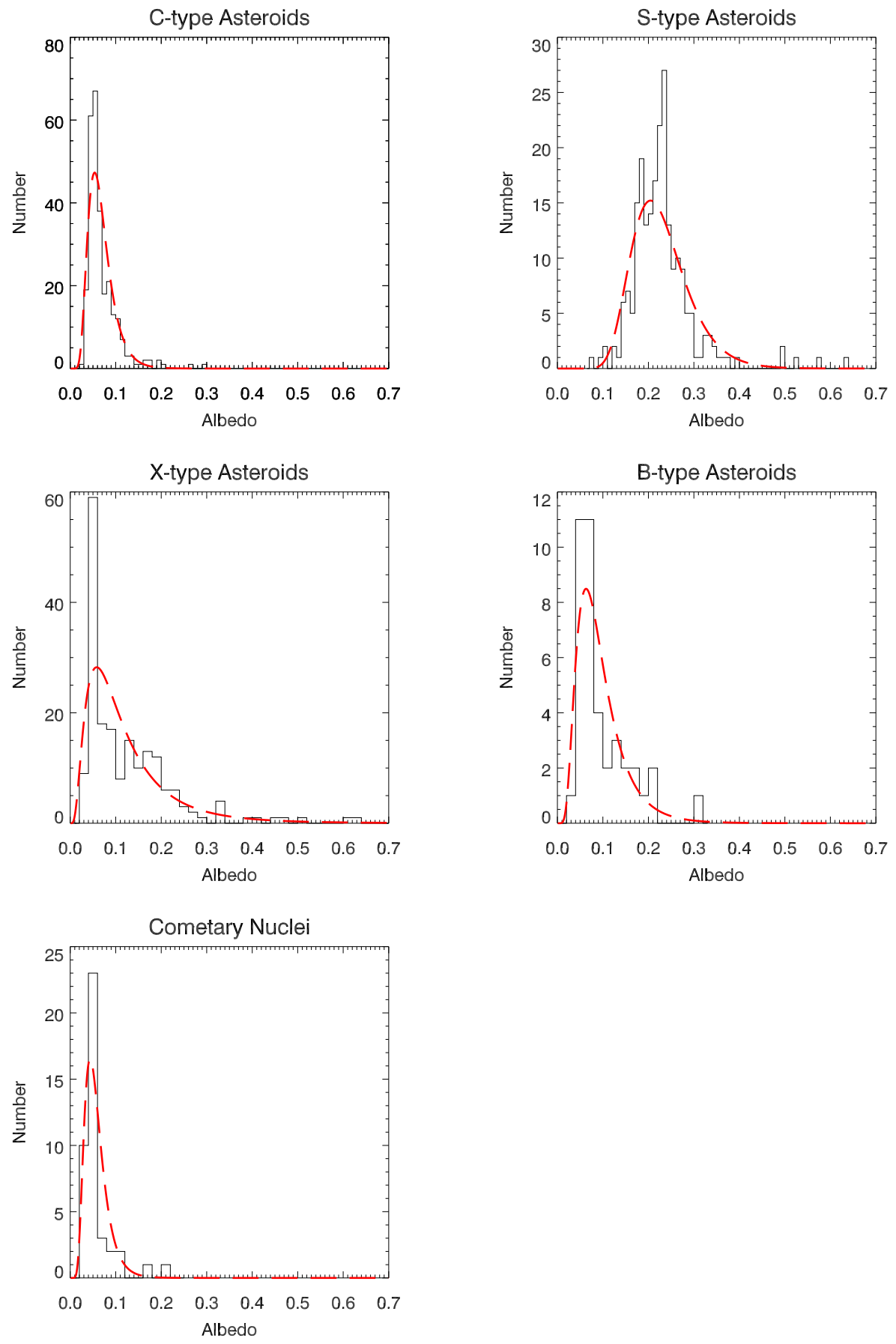

Fig. 2.- Albedo distribution of C-type, S-type, X-type, B-type, cometary nuclei (including D-type asteroids) (Usui et al. 2011). Black solid lines are histograms for the given types. Red dashed lines are the lognormal distributions calculated from the mean and standard deviation of logarithms. 

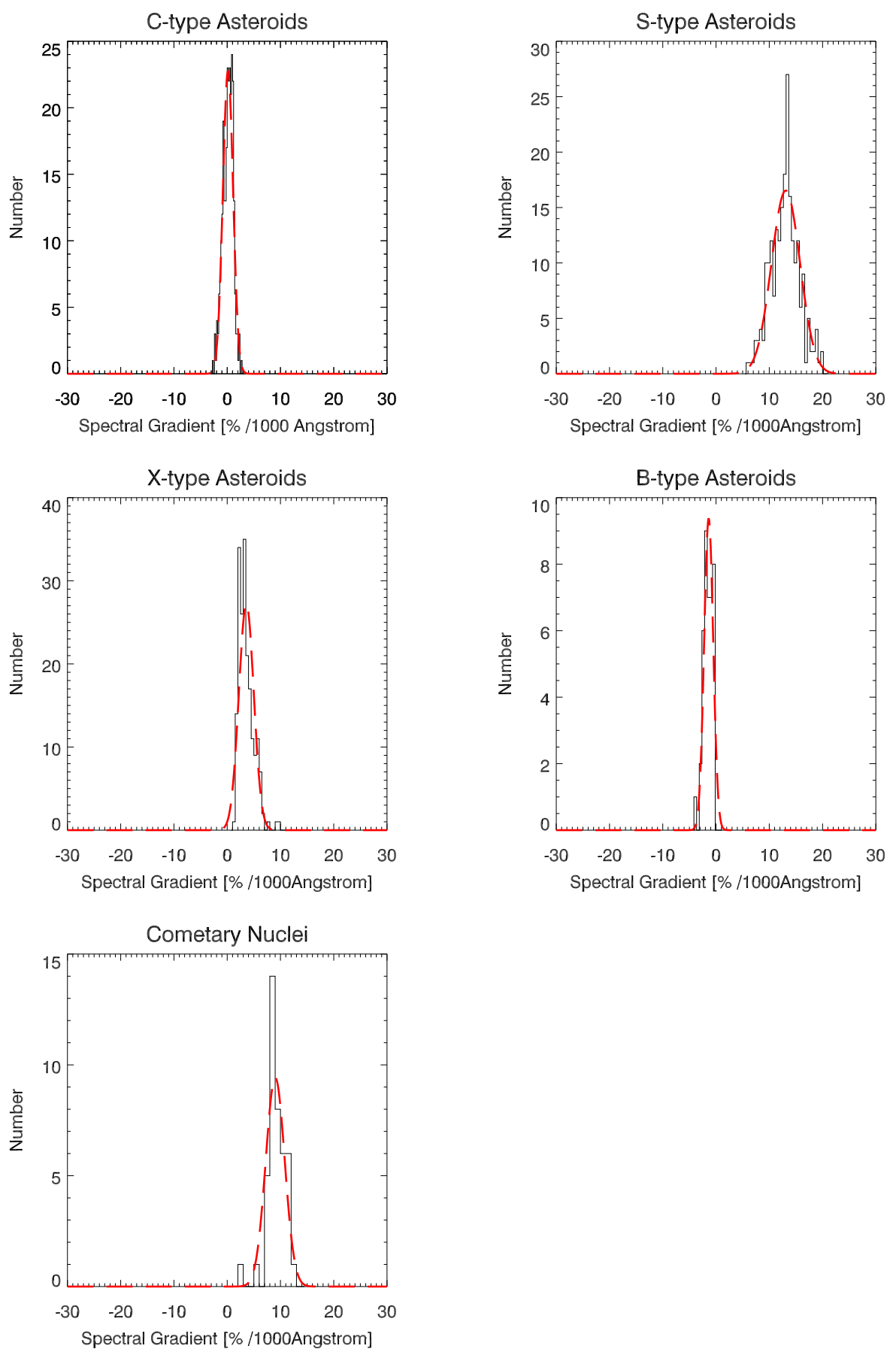

Fig. 3.- Spectral gradient distribution of C-type, S-type, X-type, B-type, cometary nuclei (including D-type asteroids) (Bus 1999; Bus \& Binzel 2002b). Black solid lines are histograms for the given types. Red dashed lines are the Gaussian distributions calculated from the mean and standard deviation. 


\section{Results}

Table 2 shows the resulting contributions from the individual sources to the IDPs cloud. To derive the ranges (which are shown as plus and minus signs in the Table 2), we made contours with the same probability in the six dimensional grid, calculated the total probability within contours around the most probable case, and derived the range with the 68.3 $\%$ confidence interval. We found that cometary nuclei (including D-type asteroids) are the primary contributors $(\sim 94 \%)$ to the IDPs cloud as predicted in the Section 2.2. The remaining part $(\sim 6 \%)$ is originated from the $\mathrm{C}$-type, $\mathrm{X}$-type and $\mathrm{B}$-type asteroids. S-type asteroids and interstellar dust have an insignificant contribution to the IDPs $(\sim 0 \%)$. Figure 4 shows the marginalized probability distributions of the four major populations.

\section{Discussion}

\subsection{Feasibility of the method}

To assess the feasibility of our approaches described above, let us discuss the three following points.

Firstly, we should consider the validity of the source populations. There are a wide variety of objects in the solar system, however, only six types of sources (five types of asteroids, comets and interstellar dust particles) are considered in this paper. Recently, the mass fraction from different taxonomic types of asteroids was studied using new multi-filter photometric survey data. DeMeo \& Carry (2013) suggested that C-type asteroids account for more than $50 \%$ of the mass in the main belt. Although S-types, P-types, B-types, and

Table 2. Contribution of 6 source populations to the IDPs

\begin{tabular}{cc}
\hline \hline Population & contribution \\
\hline Cometary nuclei & $94_{-26}^{+6} \%$ \\
B-type asteroids & $4_{-4}^{+18 \%}$ \\
X-type asteroids & $2_{-2}^{+24 \%}$ \\
C-type asteroids & $0_{-0}^{+26 \%}$ \\
S-type asteroids & $0_{-0}^{+10 \%}$ \\
Interstellar dust & $0_{-0}^{+2} \%$ \\
\hline
\end{tabular}



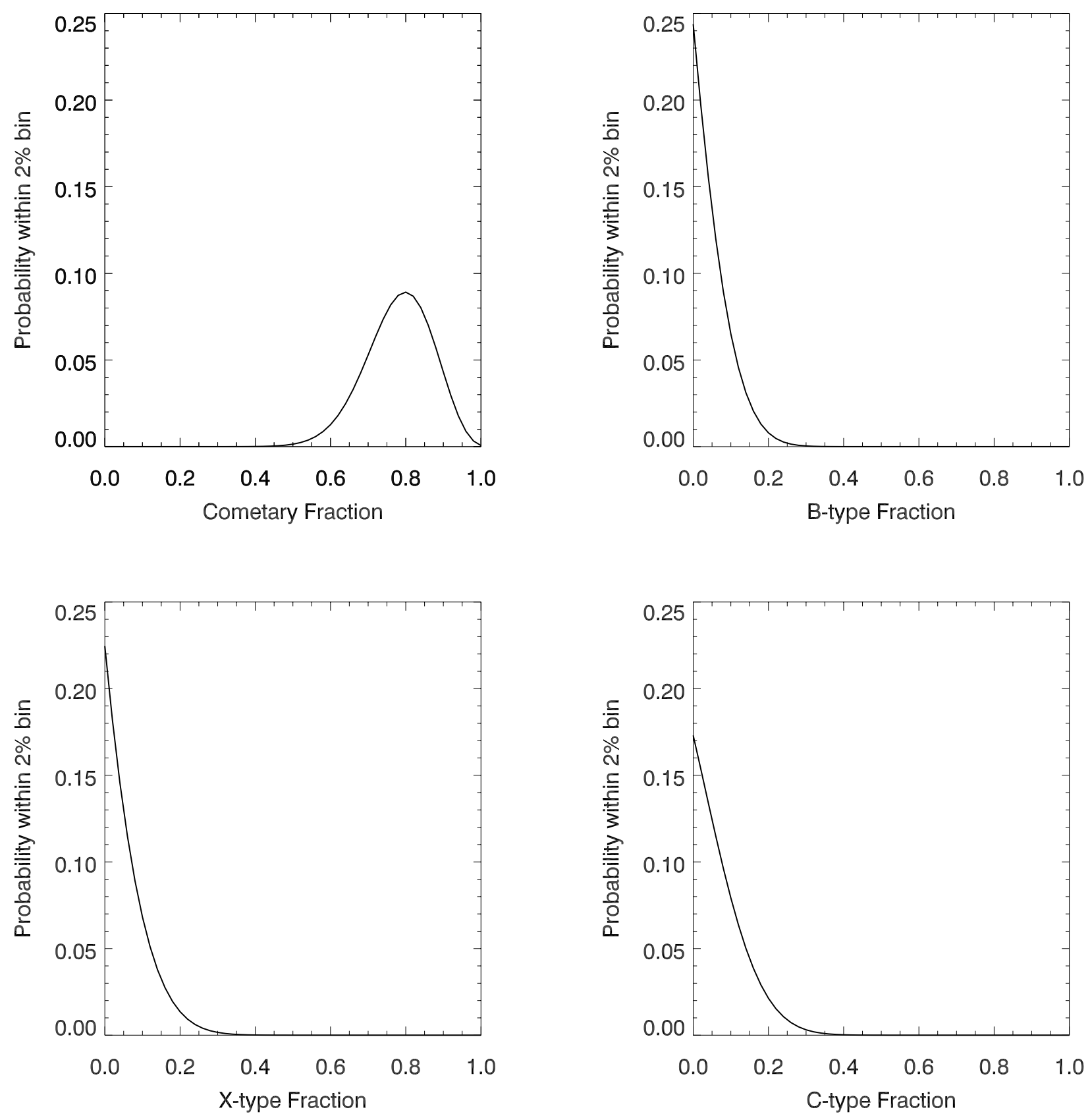

Fig. 4.- (a) - (d) Marginalized probability for the fractions about cometary (D-type asteroids), $\mathrm{B}$-type, $\mathrm{X}$-type and $\mathrm{C}$-type asteroids. The probability for the vertical axis is the values integrated over $2 \%$ bin. 
V-types have moderate fractions ( 10\% each of the total mass of asteroids in the main belt), other asteroids such as $\mathrm{K}$-types, $\mathrm{L}$-types and $\mathrm{A}$-types only have minor contributions of $<1 \%$. $\mathrm{P}$-types are included in $\mathrm{X}$-types in our assumption. Thus, we considered all but one, namely $\mathrm{V}$-types, major asteroids in this paper. We conjecture that $\mathrm{V}$-types cannot contribute to the IDPs cloud because they have very large albedos $(A=0.30$, Usui et al. (2011)). In addition, the mass fraction of $\mathrm{V}$-types is very small $(0.01 \%)$ when we exclude the largest objects in the taxonomic type (i.e. (4) Vesta). Meanwhile, the photopolarimeters on the Pioneer 10 and 11 spacecrafts revealed that the zodiacal light brightness is negligible beyond 3.3 AU (Toller 1981). Those observations suggest that the contribution from outer objects such as the Kuiper-belt objects (KBOs) may not be as large as those from asteroids, when we think about the previous dynamic studies pointing out that the dust particles from KBOs have peak densities outside of the Jovian orbits (Poppe \& Horánvi 2012; Vitense et al. 2014). The optical properties of the Centaurs show bimodality. Inactive Centaurs show ultrared spectra similar to KBOs, and active Centaurs have colors and albedos similar to cometary nuclei (Stansberry et al. 2008; Melita \& Licandro 2012). In this paper, inactive ultrared Centaurs were ignored along with KBOs and active Centaurs were treated as cometary nuclei. Some cometary nuclei have optical properties that are different from those of D-type asteroids, as in the cases of 95P/Chiron and 107P/Wilson-Harrington. We did not include these kinds of objects in this study. We do not know how many such objects exist, but these non D-type asteroidal nuclei are similar to other kinds of asteroidal groups in terms of optical properties. Therefore each population of an asteroidal group should be understood to include possible cometary nuclei whose optical properties are similar to the group. If we subdivide the $\mathrm{X}_{-}$ type asteroids into E-type, $\mathrm{M}$-type and $\mathrm{P}$-type, the results remains same, with only the confidence interval become worsen, because the optical properties of $\mathrm{P}$-type asteroids are similar to those of D-type asteroids.

Secondly, we should consider the time-evolution of the optical properties via space weathering. We assumed that the optical properties of dust particles resemble those of the source objects. However, it may not be true in some populations. Since the PoyntingRobertson lifetime of silicaceous dust particles of $1 \mathrm{~mm}$ size is about $2 \times 10^{7}$ years when released into circular orbit from 2.5AU (Mann et al. 2006) while the time scale of the space weathering is more than an order of magnitude shorter than the lifetime (Shestopalov et al. 2013, $\sim 7 \times 10^{5}$ years for S-type asteroids.), it is reasonable to assume that surfaces on both silicaceous IDPs and $\mathrm{S}$-type asteroids are altered by space weathering and therefore have similar optical properties. However, the space weathering of C-type, X-type and Btype asteroids are not well known, although there are studies, e.g. Moroz et al. (2004). Therefore, we cannot clearly discuss the optical surface maturation of IDPs originated from these asteroids. Furthermore, cometary dust particles remain within the interplanetary space 
longer than the active lifespan of cometary nuclei (Levison \& Duncan 1997, 12,000 years for the ecliptic comets), therefore the relation between the optical properties of cometary dust particles and the surfaces of active cometary nuclei is not direct. If we regard the cometary nuclei and D-type asteroids as identical, there are studies that imply that the spectra of Dtype asteroids would not change significantly over time. D-type asteroids were found in the inner main belt (DeMeo et al. 2014), and Phobos, possibly captured D-type asteroids, have optical properties of a D-type asteroid after remaining in the inner solar system for billions of years (Pajola et al. 2013, 2014). Even though these objects have albedo values slightly higher than the average of D-type asteroids, their albedos and spectral gradients are still in the range of D-type asteroids. In other direction, according to the laboratory experiments on the Targish Lake meteorites, which have spectra similar to D-type asteroids, the continuum spectrum changed to the bluer direction after being exposed to laser radiation (Hiroi \& Sasaki 2012). If these results can be applied in our case, the contribution of cometary nuclei would increase. If we think about the even now dominant cometary contribution, we can conclude that this assumption does not alter the conclusion of this paper.

Thirdly, we should consider the effects of simplification in this study. We assumed that the optical properties are randomly dispersed within a population, however this may not be true. As shown in Usui et al. (2013), there is a relation between orbital elements and optical properties. We want to emphasize that the differences in the optical properties between different types of sources are an order of magnitude bigger than the differences between sub-groups of different types of sources, as shown in Figure 1. Furthermore, we ignored the weak correlation between the albedo and spectral gradient. The relation was non-negligible for B-type asteroids. There is a possibility that the interstellar dust, which entered the solar system, has a different composition compared to the average dust particles in the Milky Way galaxy (Mann 2010), but we ignored this possibility. However, we want to justify these simplifications because the contributions from B-type asteroids and interstellar dust are almost negligible. The SMASSII catalogue is not bias-free (Mothé-Diniz et al. 2003), and we do not know the optical properties of unbiased populations, but we ignored the effect from bias. We hope that the large measurement uncertainties in the optical properties of zodiacal light cover the consequences of the bias.

\subsection{Comparison with IDP samples}

IDPs are nowadays collected in the Antarctic ice or the stratosphere around $20-25 \mathrm{~km}$ altitude using aircrafts, and they are well studied through laboratory investigations (Brownlee 1985; Engrand \& Maurette 1998). Because such particles should contribute to the zodiacal 
light before they arrive on Earth, it is important to compare our result to IDPs samples. It is known that there are two major IDPs groups, which are referred to as "chondritic smooth" (CS) and "chondritic porous" (CP). CS IDPs are composed of low porosity materials, predominantly hydrated layer silicates (Sandford \& Walker 1985). CP IDPs have large porosities of about $\sim 70 \%$. CP IDPs are dominated by anhydrous minerals. It is likely that CP IDPs originate from comets on the ground of mineralogical and petrographical properties (Bradley 2003). When the Earth passed through the dust stream of 26P/Grigg-Skjellerup (one of the JFCs), it was expected that 1-50\% of the total collected dust larger than 40 $\mu \mathrm{m}$ could originate from that comet (Messenger 2002). These were actually CP type IDPs with primitive anhydrous composition, supporting the assumption of that CP IDPs are of cometary origin (Busemann et al. 2009). CS IDPs are thought to be derived from primitive (not differentiated) asteroids, because comets do not exhibit spectral signatures of hydrated silicate while asteroids do (McAdam et al. 2015).

Bradley et al. (1996) measured the reflectance spectra of IDPs samples in the optical wavelength and found that CS IDPs generally exhibit flat spectra with weak fall-off from $6000 \AA$ towards $8000 \AA$ (similar to CI and CM meteorites or C-type asteroids with $S^{\prime} \sim 0$ ) whereas CP IDPs exhibit upward spectra $\left(S^{\prime}>0\right)$ without remarkable curvature, although these IDP samples have a variety of albedo values and spectral slopes. Figure 5 compares the synthesized spectrum of zodiacal light based on our mixing model of small bodies with those of most typical CS IDPs (W7040A15) and CP IDPs (W030A5) (Bradley et al. 1996). We also show the input spectrum in Figure 5, which was obtained from a multi-band photometry in Leinert et al. (1998) and anti-solar point observation (Ishiguro et al. 2013). The synthesized spectrum of zodiacal light is similar to that of CP IDPs (W030A5) in that it shows a low albedo value and positive slope $\left(S^{\prime}>0\right)$ but it is different from that of CS IDPs in that it does not show a positive slope beyond $\sim 6500 \AA$. It should be noted that the fall-offs below $4500 \AA$ in IDPs signals are artifacts of the measurements caused by small size effects and should be ignored for the comparison (Bradley et al. 1996). The spectral similarity leads to our assumption that the interplanetary dust complex is dominated by CP IDPs (i.e. dominance of cometary particles in zodiacal cloud). It is also curious to notice that CP IDPs tend to have cluster structures of 20-100 $\mu \mathrm{m}$ (Bradley et al. 1996). The size is in accordance with the effective size of zodiacal light dust particles evaluated by the IDPs size distribution model (Grun et al. 1985) .

There seems to be a difference on the quantitative estimates of IDPs origins between laboratory investigations of IDPs samples and ours. Bradley (2003) studied 200 chondritic IDPs from the stratosphere and found that about a half of them are classified into CP IDPs. Similarly, Noguchi et al. (2015) investigated micrometeorite samples in Antarctica and suggested $\sim 25 \%$ or even less are categorized into CP IDPs. Whereas we acknowledge 


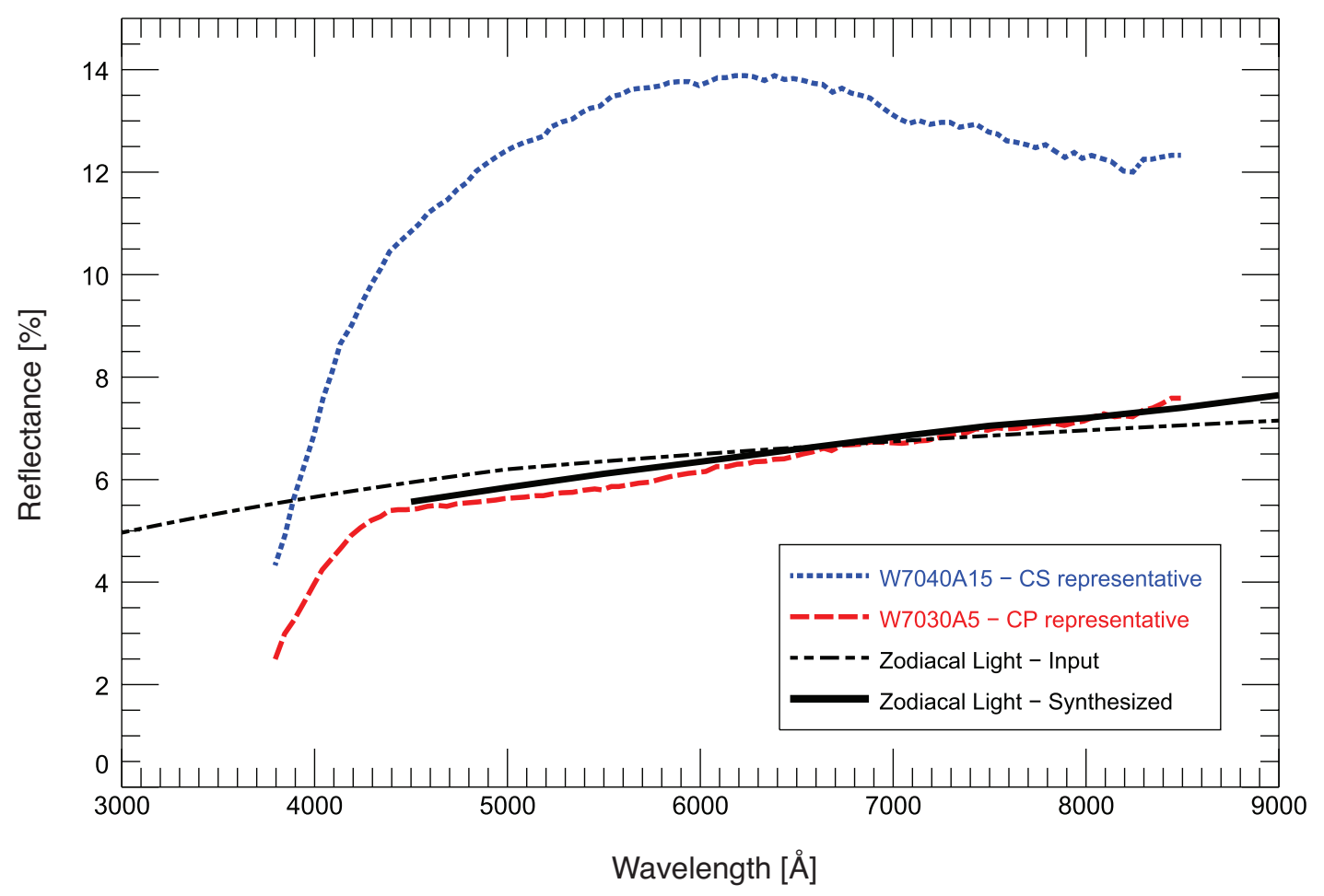

Fig. 5.- Comparisons of spectra between our synthesized zodiacal light model (thick continuous line), CS and CP IDPs (dotted and dashed lines) (Bradley et al. 1996). The observed reference spectrum of zodiacal light is also shown (see Section 2.1). Note the drop-offs in the IDP spectra at less than $4000 \AA$ artifacts. 
that these laboratory investigations provide reliable results regarding the fraction of CS and CP IDPs fallen to Earth, we would draw attention to the sampling bias of the laboratory studies of IDPs. Asteroidal dust particles could be collected on Earth more selectively than cometary dust particles because of the orbital properties. The impact cross-section of asteroidal dust particles to the Earth is a few times larger than that of cometary ones. Furthermore, the impact cross-section of the Earth can be a few 1000 times larger for dust particles trapped in quasi-satellite resonance, which favors asteroidal particles (Kortenkamp $2013)$.

\subsection{Comparison with previous studies}

Our results are in agreement with kinematic, dynamical studies based on spatial distribution of zodiacal light. Numerical simulations of Nesvorný et al. (2010) concluded that $\gtrsim 90 \%$ of the zodiacal emission comes from JFC originated IDPs, and $\lesssim 10 \%$ from the Oort cloud comets or asteroids. These results coincide with our results of $>90 \%$ cometary contribution. However, we could not optically distinguish different sub-populations of comets in this study. Therefore, we cannot know how large of a fraction of cometary IDPs originated from JFCs.

We compared our results with infrared spectroscopic observations of zodiacal light. By using mean albedo values and contributions from this study and adopting a typical visualinfrared spectrum of the populations from Bus \& Binzel (2002a), we synthesized the model spectrum of zodiacal light, and extrapolated it to the near-infrared wavelength, shown in Figure 6. At wavelengths shorter than $1.6 \mu \mathrm{m}$, the observed NIR spectra of the zodiacal light (Tsumura et al. 2010) are similar to the synthesized spectra in large-scale, but have absorption-like dark wavelengths around 1.3-1.4 $\mu \mathrm{m}$. In this wavelength regime, the spectra of average B-type, C-type and X-type asteroids are bluer than those of zodiacal light, as expected in this study. The observed spectra have large uncertainties at wavelengths longer than $1.6 \mu \mathrm{m}$. It is, therefore, hard to conclude clearly, but the observed spectra are closer to those of D-type, C-type or X-type asteroids than to those of S-type or B-type asteroids. Overall, the synthesized spectrum in this work is consistent with the observed spectrum from rocket-borne observations (Matsuura et al. 1995; Tsumura et al. 2010) and recent space observations of AKARI (Tsumura et al. 2013), but it does not agree with the space observation of IRTS (Matsumoto et al. 1996). The observed NIR spectra of zodiacal light are similar to those of D-type asteroids or cometary nuclei, which is in agreement with this work. Keeping in mind that we applied extrapolation through the model from optical observation, this match in the NIR reflected spectrum supports our conclusion of dominating 
cometary contribution.

We wish to acknowledge Dr. Fumihiko Usui for his valuable comments to the manuscript, and Dr. Kohji Tsumura for the kind offering of his data. This work was supported by the National Research Foundation of Korea (NRF) funded by the South Korean government (MEST) (Grant No. 2012R1A4A1028713).

\section{REFERENCES}

Abell, P. A., Fernández, Y. R., Pravec, P., et al. 2005, Icarus, 179, 174

A’Hearn, M. F., Campins, H., Schleicher, D. G., \& Millis, R. L. 1989, ApJ, 347, 1155

Binzel, R. P., Rivkin, A. S., Stuart, J. S., et al. 2004, Icarus, 170, 259

Boehnhardt, H., Tozzi, G. P., Bagnulo, S., et al. 2008, A\&A, 489, 1337

Bradley, J. P. 2003, Treatise on Geochemistry, 1, 689

Bradley, J. P., Keller, L. P., Brownlee, D. E., \& Thomas, K. L. 1996, Meteoritics and Planetary Science, 31, 394

Brownlee, D. E. 1985, Annual Review of Earth and Planetary Sciences, 13, 147

Buffington, A., Bisi, M. M., Clover, J. M., et al. 2009, Icarus, 203, 124

Bus, S. J. 1999, PhD thesis, Massachusetts Institute of Technology

Bus, S. J., \& Binzel, R. P. 2002a, Icarus, 158, 146

-. 2002b, Icarus, 158, 106

Busemann, H., Nguyen, A. N., Cody, G. D., et al. 2009, Earth and Planetary Science Letters, 288, 44

Campins, H., A’Hearn, M. F., \& McFadden, L.-A. 1987, ApJ, 316, 847

Campins, H., \& Fernández, Y. 2002, Earth Moon and Planets, 89, 117

Campins, H., Licandro, J., Pinilla-Alonso, N., et al. 2007, AJ, 134, 1626

Campins, H., Ziffer, J., Licandro, J., et al. 2006, AJ, 132, 1346 


\section{Zodiacal light spectrum}

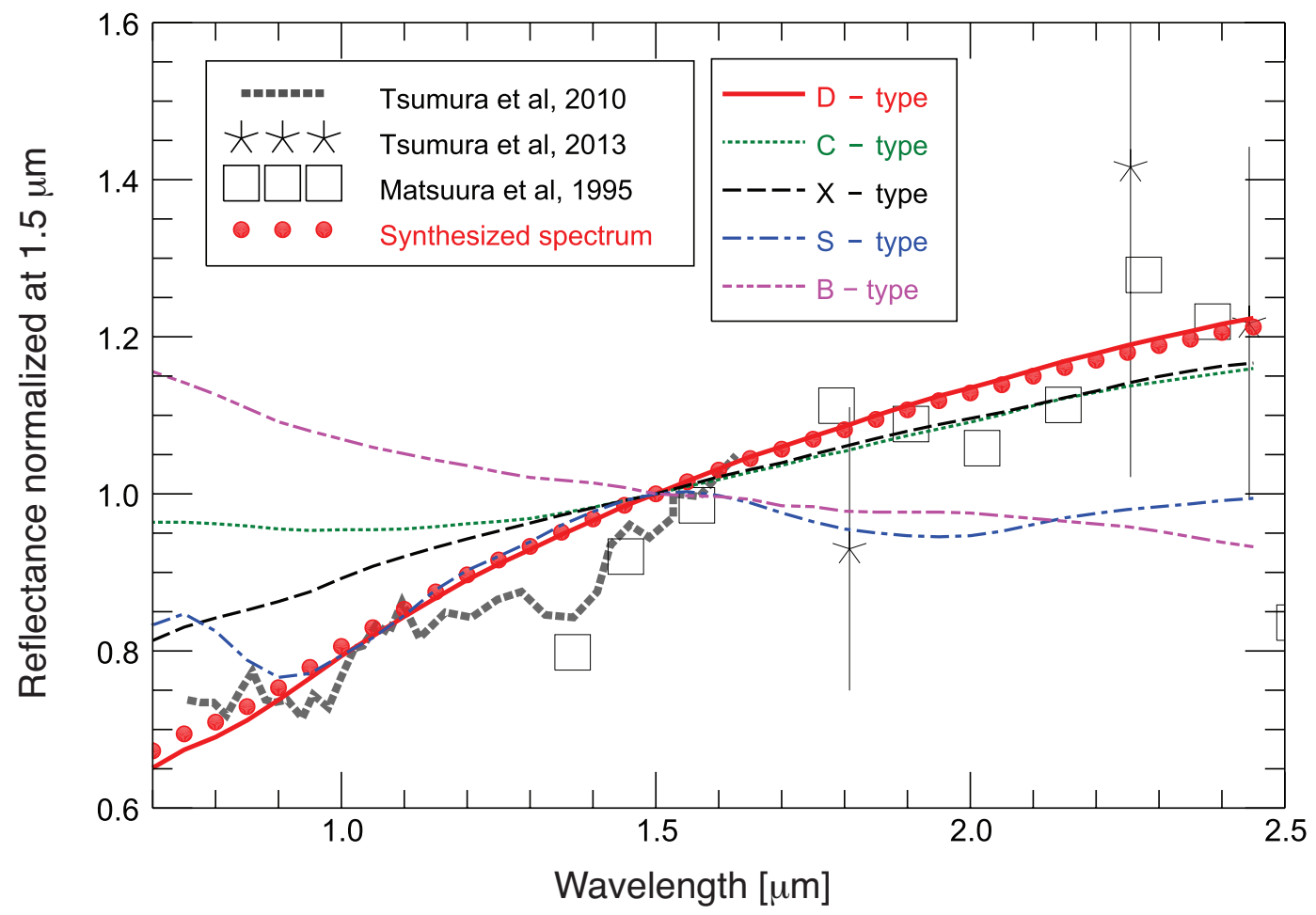

Fig. 6.- Comparison of near-infrared zodiacal light spectra between our synthesized model and observed data (Matsuura et al. 1995; Tsumura et al. 2010, 2013). The S-220-11 rocket data at an ecliptic latitude of $10^{\circ}$ from Matsuura et al. (1995) was used in the figure. The template spectra of each type of asteroid are from Bus \& Binzel (2002a), and the solar spectra are from Guevmard (2004). The templates, synthesized spectra, and the data from Tsumura et al. (2010) are normalized at $1.5 \mu \mathrm{m}$, and the data from (Matsuura et al. 1995; Tsumura et al. 2013) are scaled to match our model spectrum at 1.8-2.5 $\mu \mathrm{m}$. 
DeMeo, F. E., Binzel, R. P., Carry, B., Polishook, D., \& Moskovitz, N. A. 2014, Icarus, 229, 392

DeMeo, F. E., \& Carry, B. 2013, Icarus, 226, 723

Dermott, S. F., Grogan, K., Gustafson, B. A. S., et al. 1996, in Astronomical Society of the Pacific Conference Series, Vol. 104, IAU Colloq. 150: Physics, Chemistry, and Dynamics of Interplanetary Dust, ed. B. A. S. Gustafson \& M. S. Hanner, 143

Dikarev, V., Grün, E., Landgraf, M., Baggaley, W. J., \& Galligan, D. P. 2001, in ESA Special Publication, Vol. 495, Meteoroids 2001 Conference, ed. B. Warmbein, 609-615

Divine, N. 1993, J. Geophys. Res., 98, 17029

Draine, B. T. 2003, ApJ, 598, 1017

Dumont, R., \& Levasseur-Regourd, A.-C. 1988, A\&A, 191, 154

Engrand, C., \& Maurette, M. 1998, Meteoritics and Planetary Science, 33, 565

Fernández, Y. R., Campins, H., Kassis, M., et al. 2006, AJ, 132, 1354

Fernández, Y. R., Lisse, C. M., Ulrich Käufl, H., et al. 2000, Icarus, 147, 145

Fixsen, D. J., \& Mather, J. C. 2002, ApJ, 581, 817

Gor'kavyi, N. N., Ozernoy, L. M., \& Mather, J. C. 1997, ApJ, 474, 496

Grun, E., Zook, H. A., Fechtig, H., \& Giese, R. H. 1985, Icarus, 62, 244

Gueymard, C. A. 2004, Solar Energy, 76, 423

Hahn, J. M., Zook, H. A., Cooper, B., \& Sunkara, B. 2002, Icarus, 158, 360

Hanner, M. S. 1980, Icarus, 43, 373

Hiroi, T., \& Sasaki, S. 2012, LPI Contributions, 1667, 6109

Ishiguro, M., Yang, H., Usui, F., et al. 2013, ApJ, 767, 75

Jewitt, D., \& Luu, J. 1989, AJ, 97, 1766

Keller, H. U., Delamere, W. A., Reitsema, H. J., Huebner, W. F., \& Schmidt, H. U. 1987, A\&A, 187, 807

Kelley, M. S., Wooden, D. H., Tubiana, C., et al. 2009, AJ, 137, 4633 
Kelsall, T., Weiland, J. L., Franz, B. A., et al. 1998, ApJ, 508, 44

Kortenkamp, S. J. 2013, Icarus, 226, 1550

Lamy, P. L., Toth, I., Groussin, O., et al. 2008, A\&A, 489, 777

Lamy, P. L., Toth, I., Weaver, H. A., et al. 2006, A\&A, 458, 669

Leinert, C., Bowyer, S., Haikala, L. K., et al. 1998, A\&AS, 127, 1

Levison, H. F., \& Duncan, M. J. 1997, Icarus, 127, 13

Li, J.-Y., A'Hearn, M. F., Belton, M. J. S., et al. 2007, Icarus, 187, 41

Li, J.-Y., Besse, S., A'Hearn, M. F., et al. 2013, Icarus, 222, 559

Lumme, K., \& Bowell, E. 1985, Icarus, 62, 54

Luu, J., \& Jewitt, D. 1990, Icarus, 86, 69

Luu, J. X. 1993, Icarus, 104, 138

Mann, I. 2010, ARA\&A, 48, 173

Mann, I., \& Czechowski, A. 2005, ApJ, 621, L73

Mann, I., Köhler, M., Kimura, H., Cechowski, A., \& Minato, T. 2006, A\&A Rev., 13, 159

Matsumoto, T., Kawada, M., Murakami, H., et al. 1996, PASJ, 48, L47

Matsuura, S., Matsumoto, T., Matsuhara, H., \& Noda, M. 1995, Icarus, 115, 199

McAdam, M. M., Sunshine, J. M., Howard, K. T., \& McCoy, T. M. 2015, Icarus, 245, 320

Melita, M. D., \& Licandro, J. 2012, A\&A, 539, A144

Messenger, S. 2002, Meteoritics and Planetary Science, 37, 1491

Millis, R. L., A’Hearn, M. F., \& Campins, H. 1988, ApJ, 324, 1194

Moroz, L., Baratta, G., Strazzulla, G., et al. 2004, Icarus, 170, 214

Mothé-Diniz, T., Carvano, J. M. á., \& Lazzaro, D. 2003, Icarus, 162, 10

Nesvorný, D., Jenniskens, P., Levison, H. F., et al. 2010, ApJ, 713, 816 
Noguchi, T., Ohashi, N., Tsujimoto, S., et al. 2015, Earth and Planetary Science Letters, 410,1

Ootsubo, T., Ueno, M., Ishiguro, M., et al. 2009, in Astronomical Society of the Pacific Conference Series, Vol. 418, AKARI, a Light to Illuminate the Misty Universe, ed. T. Onaka, G. J. White, T. Nakagawa, \& I. Yamamura, 395

Pajola, M., Lazzarin, M., Dalle Ore, C. M., et al. 2013, ApJ, 777, 127

Pajola, M., Lazzarin, M., Bertini, I., et al. 2014, Memorie della Societa Astronomica Italiana Supplementi, 26, 67

Poppe, A. R., \& Horányi, M. 2012, Geophys. Res. Lett., 39, 15104

Reach, W. T., Morris, P., Boulanger, F., \& Okumura, K. 2003, Icarus, 164, 384

Sagdeev, R. Z., Blamont, J., Galeev, A. A., et al. 1986, Nature, 321, 259

Sandford, S. A., \& Walker, R. M. 1985, ApJ, 291, 838

Shestopalov, D. I., Golubeva, L. F., \& Cloutis, E. A. 2013, Icarus, 225, 781

Stansberry, J., Grundy, W., Brown, M., et al. 2008, Physical Properties of Kuiper Belt and Centaur Objects: Constraints from the Spitzer Space Telescope, ed. M. A. Barucci, H. Boehnhardt, D. P. Cruikshank, A. Morbidelli, \& R. Dotson, 161-179

Thomas, N., \& Keller, H. U. 1989, A\&A, 213, 487

Toller, G. N. 1981, PhD thesis, State Univ. of New York, Stony Brook (USA)

Tsumura, K., Matsumoto, T., Matsuura, S., et al. 2013, PASJ, 65, 119

Tsumura, K., Battle, J., Bock, J., et al. 2010, ApJ, 719, 394

Tubiana, C., Barrera, L., Drahus, M., \& Boehnhardt, H. 2008, A\&A, 490, 377

Usui, F., Kasuga, T., Hasegawa, S., et al. 2013, ApJ, 762, 56

Usui, F., Kuroda, D., Müller, T. G., et al. 2011, PASJ, 63, 1117

Vitense, C., Krivov, A. V., \& Löhne, T. 2014, AJ, 147, 154 